\title{
Interobserver reliability in measuring flexion, internal rotation, and external rotation of the hip using a plurimeter
}

\author{
P R Croft, E S Nahit, G J Macfarlane, A J Silman; on behalf of the Primary Care \\ Rheumatology Society Hip Study Group ${ }^{\star}$
}

\begin{abstract}
Objective-To determine reliability of the measurement of hip movements (flexion, internal rotation, and external rotation) between medical practitioners.

Methods-Six clinicians carried out measurements of hip movements on each of six patients with osteoarthritis of one hip, using a specifically designed plurimeter.

Results-There was no evidence of any systematic difference between medical practitioners in the measurement of hip flexion, internal rotation, or external rotation. The degree of agreement was greatest for hip flexion.

Conclusions-This study has shown that measurement of range of movement at the hip is repeatable between practitioners using a simple plurimeter and may represent an examination that is suitable for monitoring progress and treatment.
\end{abstract}

(Ann Rheum Dis 1996; 55: 320-323)

Little is known about the natural history of hip osteoarthritis in its early development, or about the stage at which patients with this disease present to their general practitioner. We need to know more about it in order to investigate the benefits of early treatment, the potential prevention of progression, and the optimal timing of surgical intervention. Such studies should be carried out in primary care, but standard measures of disease severity need to be established in order to monitor change over time within and between practices.

The tool used traditionally to measure osteoarthritis of the hip is the radiograph. The cardinal features of joint space narrowing, sclerosis, and osteophytosis reflect the underlying processes of cartilage loss, bony thickening, and new bone formation. From the patient's perspective, however, it is symptoms that are important, and up to $40 \%$ of people with definite radiographic changes of hip osteoarthritis do not report pain in the hip. ${ }^{1}$ Self reporting of pain severity and restriction of daily activity thus provides one means to measure progress and outcome. However, another approach that might complement such subjective assessments is to measure the range of movement at the hip joint.

Orthopaedic clinical texts indicate that, as osteoarthritis of the hip progresses, the movements at the joint become increasingly restricted. ${ }^{2}$ There is some disagreement about the most sensitive indicator of change, though internal rotation is generally considered to be the first to deteriorate, followed by external rotation and flexion. ${ }^{2}$ However, there is virtually no information about the performance of these tests in terms of their repeatability between different observers. Standard goniometry is difficult to adapt to measure hip rotation, and alternative simple methods are required.

We have therefore carried out a study in which doctors measured movement in the hips of patients with and without osteoarthritis, to estimate the extent of observer variation and to evaluate the performance of a fluid filled plurimeter in recording hip movements.

\section{Patients and methods}

Six outpatients, all of whom had symptomatic osteoarthritis of one hip only and no arthroplasty, volunteered to participate in the study.

Six clinicians, including five general practitioner members of the Primary Care Rheumatology Society and a hospital physician, carried out the measurements. There was a short training session in which agreement was reached between the participants on the method of measuring each hip movement. The instrument used in the experiment was a fluid filled plurimeter designed by $\mathrm{Dr}$ Jules Rippstein (Switzerland), who had constructed a special footplate for the purpose of the hip measurements (fig 1A). The plurimeter has a rotating dial, which allows easy reading of the angle of movement to the nearest $2^{\circ}$.

Each clinician examined both hips of each patient once. In each hip, the range of passive flexion, passive external rotation, and passive internal rotation was measured and recorded by the clinician. The order of examination of the movements in each patient by the clinicians was determined by a randomised Latin Square design to ensure that any effect of order on the measurements could be assessed.

Flexion was measured with the patient supine and with assistance from the examining clinician to reach maximum flexion or the point of restriction by pain. The lower leg was maintained parallel to the bed and the plurimeter (fig 1A) was placed on the anterior or lateral thigh. Both the neutral position (fig $1 \mathrm{~B})$ and the position of maximum flexion (fig 
1C) were recorded. It had previously been established that readings were unaffected by the anterior or lateral placement of the plurimeter. Rotation was measured with the patient sitting with the legs flexed at the knee and the lower legs unsupported over the edge of the bed. A plurimeter recording was made in this 'neutral' position (fig 1D) with the instrument placed on the lateral or medial calf in the vertical plane as, again, it had been established that the alternative positions did not affect the measurement. The extent of movement laterally (internal rotation) (fig $1 \mathrm{E}$ ) and medially (external rotation) (fig $1 \mathrm{~F}$ ), was then recorded, with assistance from the examiner to reach the point of maximal rotation or at which pain restricted further movement.

\section{STATISTICAL ANALYSIS}

Two elements of observer variability were examined: ${ }^{3}$ systematic bias and random error. The former was assessed by constructing box plots for each clinician, allowing a comparison of their medians, interquartile ranges, and any extreme values.

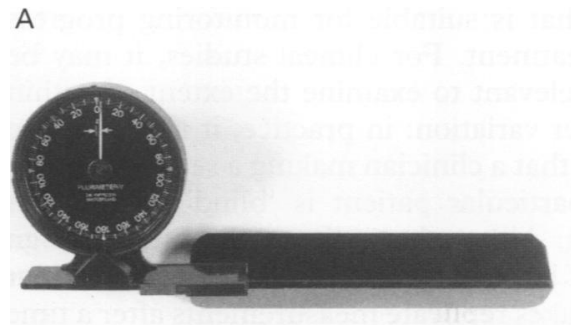

B

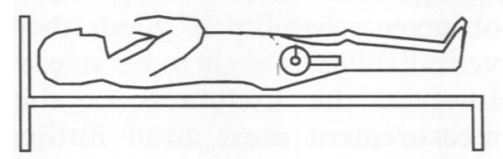

C

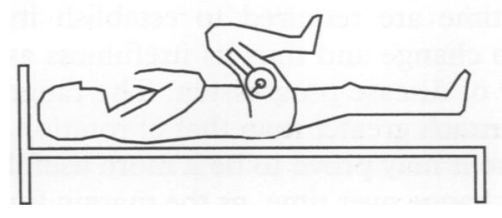

Figure 1 Hip examination and measurements. A: The plurimeter. B: Hip flexion-neutral position. C: Hip flexion-maximal position. D: Rotation-neutral position. E: Internal rotation. F: External rotation.
Analysis of variance was used to partition the variation attributable to clinician differences, to patient differences, and to an order effect. Clinician agreement for each measurementhip flexion, internal rotation, and external rotation-was analysed by considering the total amount of variation present in the measurements and determining the amount attributable to the patients $\left(\sigma_{p}^{2}\right)$, the amount attributable to the clinicians $\left(\sigma^{2}{ }_{d}\right)$, and that (any other variation determined) attributable to residual or random variation $\left(\sigma_{e}^{2}\right)$. The proportion of variation explained by the patients was termed the 'intraclass correlation coefficient' (ICC) and was calculated for each measurement:

$$
\text { ICC }=\frac{\sigma_{p}^{2}}{\sigma_{p}^{2}+\sigma_{d}^{2}+\sigma^{2}{ }_{e}}
$$

Ideally, most of the variation should be explained by patient differences. As results did not differ depending on whether diseased or non-diseased hips were considered, the results have been pooled for both hips.

All statistical analyses have been carried out using the STATA statistical package,${ }^{4}$ and for the analyses of variance, assumptions regarding the independence and the normal distribution of errors were checked. The assumption of normal distribution of errors was violated in the case of measurements relating to internal rotation; a logarithmic transformation was applied to these data and the assumption of normal distribution of errors was then satisfied.

\section{Results}

Figure 2A, B, and C, respectively, show 'box and whisker' plots of the measurements made by the six clinicians for hip flexion, internal rotation (outward movement), and external rotation (inward movement). The interquartile ranges of the distribution of readings is shown as the box, the line within is the median reading, and the bars extending out from the box represent the values of the upper and lower readings. Certain 'extreme values' (distance between the observation and median value greater than 1.5 times the interquartile range) are plotted individually as circles. Where no whisker occurs (figure 2B, clinician 4) the extreme reading is the same as the 25th centile; similarly, the median can coincide with an interquartile boundary (figure $2 \mathrm{~B}$, clinician 5 ). The distributions of the individual observers' scores were broadly similar for each of the three measurements, suggesting no major systematic bias.

The table summarises the results of the analyses of variance for hip flexion, internal rotation, and external rotation. All analyses of variance indicate strong between patient differences, as expected. The differences between clinicians did not prove significant for any of the measurements. There was no order effect for any of the measurements.

Agreement between clinicians was greatest for hip flexion (ICC $=0 \cdot 87$ ). For internal rotation and external rotation, agreement was 
lower (ICC $=0.48$ and 0.43 , respectively). Range of movement is smaller for both internal and external rotation and hence patient differences are small. This is one reason why fewer of the differences in readings for these two movements could be explained by patient differences, and why more were explained by non-systematic differences between clinicians.
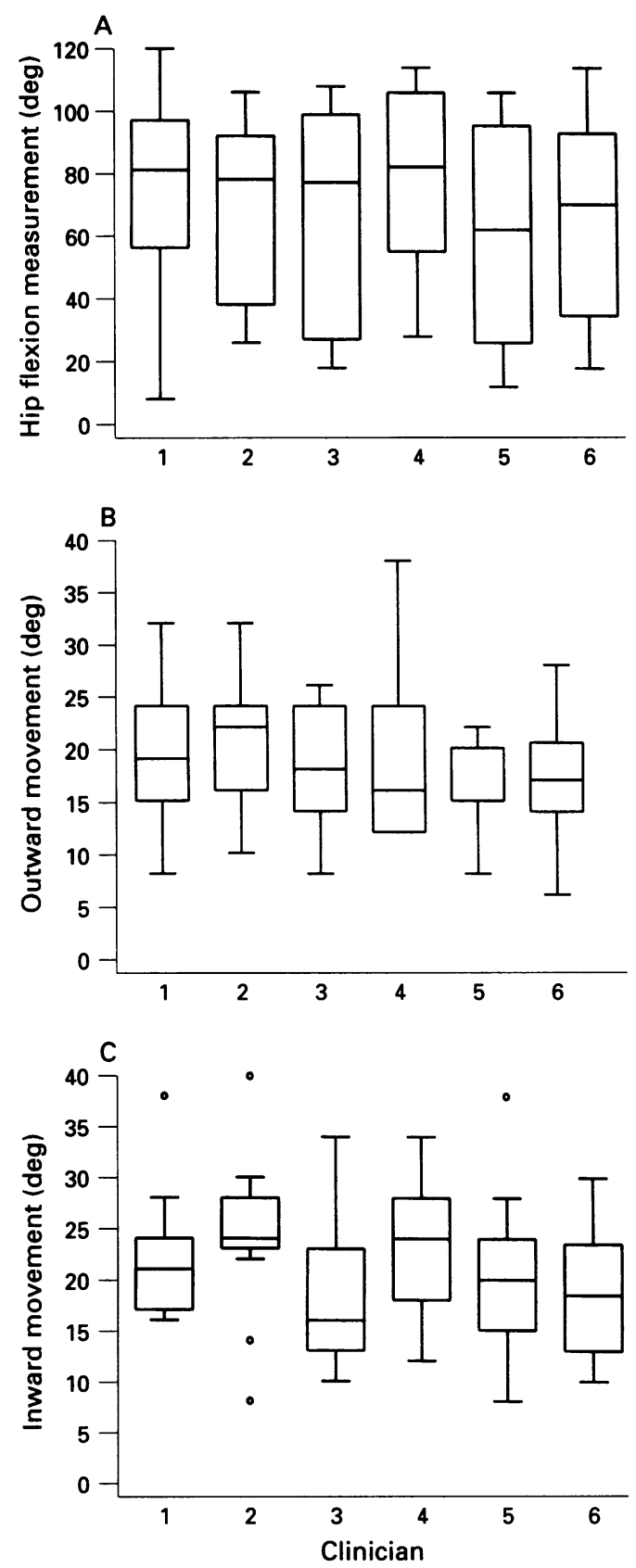

Figure 2 Box plots of the distribution of readings for each clinician. Box = interquartile ranges of the distribution of readings; internal line $=$ median reading; whisker $=$ upper and lower readings. O = Individual extreme values. A: Hip flexion. B: Internal rotation. C: External rotation.

\section{Discussion}

Osteoarthritis is the commonest cause of disability in older age groups in Britain, and involvement of the hip its most disabling form, yet generally accepted clinical criteria that would be accessible and reliable for primary care physicians to use to diagnose and measure progression of the disease, are lacking. Although pain and self reported restriction of activity are likely to be a fruitful focus of epidemiological research into outcome and progression, a simple examination of the range of movement at the hip joint would greatly enhance the general practitioner's ability to monitor progress and treatment.

The number of patients we studied was small, and there may be certain patient characteristics that might influence reliability of measurements. Although we found no difference between symptomatic and asymptomatic hips in this sample, other features such as very severe pain and obesity might influence the results obtained.

Measuring the range of hip movement is difficult with the traditional goniometer. This study has shown that the use of a simple plurimeter to measure hip movement did not result in any systematic difference between practitioners and might represent an examination that is suitable for monitoring progress and treatment. For clinical studies, it may be more relevant to examine the extent of within observer variation: in practice, it is difficult to ensure that a clinician making a second reading on a particular patient is 'blind' to the first reading. Although an alternative study design could be used in which the examiner undertakes replicate measurements after a time interval sufficiently long to prevent recall, the problem would arise that there may have been true patient change in the interval. In practice, if between observer reliability is good, then within observer reliability is likely to be at least as good. However, the usefulness of this method of measurement must await further investigations. Internal rotation is generally considered to be the earliest movement to deteriorate in hip osteoarthritis. Prospective studies to follow the change in this measurement over time are required to establish its sensitivity to change and thus its usefulness as an indicator of disease progression. The range of flexion is much greater than that of rotation, and therefore it may prove to be a more useful measure of change over time, as the margin for change is potentially so much more than with the more restricted movements of rotation. In addition, the way in which objectively measured movement at the hip relates to

Summary of results of analysis of variance

\begin{tabular}{|c|c|c|c|c|c|c|c|c|c|}
\hline \multirow[b]{2}{*}{ Source } & \multicolumn{3}{|l|}{ Hip flexion } & \multicolumn{3}{|c|}{ Internal rotation } & \multicolumn{3}{|c|}{ External rotation } \\
\hline & $M S q$ & $F$ & $p$ & $M S q$ & $F$ & $p$ & $M S q$ & $F$ & $p$ \\
\hline $\begin{array}{l}\text { Patients } \\
\text { Clinicians } \\
\text { Order } \\
\text { Residual } \\
\text { Total } \\
\text { ICC }\end{array}$ & $\begin{array}{r}10928 \cdot 1 \\
440 \cdot 7 \\
287 \cdot 2 \\
245 \cdot 9 \\
1014 \cdot 8 \\
0 \cdot 87\end{array}$ & $\begin{array}{r}44.4 \\
1.8 \\
1.2\end{array}$ & $\begin{array}{l}>0.001 \\
\text { NS } \\
\text { NS }\end{array}$ & $\begin{array}{c}170 \cdot 2 \\
20 \cdot 8 \\
42 \cdot 5 \\
27 \cdot 0 \\
37 \cdot 7 \\
0 \cdot 48\end{array}$ & $\begin{array}{l}6.3 \\
0.8 \\
1.6\end{array}$ & $\begin{array}{l}>0.01 \\
\text { NS } \\
\text { NS }\end{array}$ & $\begin{array}{c}228 \cdot 1 \\
72 \cdot 8 \\
43 \cdot 3 \\
37 \cdot 0 \\
53 \cdot 4 \\
0.43\end{array}$ & $\begin{array}{l}6 \cdot 2 \\
2 \cdot 0 \\
1 \cdot 2\end{array}$ & $\begin{array}{l}>0.001 \\
\text { NS } \\
\text { NS }\end{array}$ \\
\hline
\end{tabular}

ICC = Intraclass correlation coefficient; $\mathrm{MSq}=$ mean squares 
radiographic change and patient symptoms will be a crucial issue in determining the usefulness of measurements of the range of movement, both in research and in clinical practice.

For the present, however, we have established the between observer reliability of a simple measurement of the range of hip movements in a group of patients whose hips showed varying degrees of radiographic osteoarthritis. We are now carrying out a prospective study in primary care to establish the association of these measurements with clinical and radiological features of hip osteoarthritis and with changes in these characteristics over time.

This work was supported by the Arthritis and Rheumatism Council, United Kingdom.
*Other members of the group involved in producing this manuscript were: Drs C Cooper, G Hosie, S Bradbury, M Hall, A Vaghmaria, E Hay, and N Cheung.

The authors are grateful to Joanna Schollum, Ann Papageorgiou, Lesley Jordan, Daniel Pope, Elaine Thomas, the Haywood Hospital nursing and administrative staff who helped in the study, the patients who participated, and to Dr Jules Rippstein for his participation and advice during the may be obtained from Dr Rippstein at: Pierrettes 53, CH-1093 may be obtained from Dr Rippstein at: Pierrettes 53,

1 US Department of Health, Education and Welfare. Basic data on arthritis: knee, hip and sacroiliac joints in adult ages 25-74 years. Publication No 79-1661, Series 11, No 213 . Washington DC: DHEW, 1971-1975.

2 Hoppenfeld S. Physical examination of the spine and extremities. New York: Appleby-Century-Crofts, 1976.

3 Brennan P, Silman A, Black C, et al. Reliability of skin involvement measures in scleroderma. $\mathrm{Br} \mathcal{F}$ Rheumatol 1992; 31: 457-60.

4 StataCorp. Stata statistical software: release 4.0. College Station, TX: Stata Corporation, 1995. 